\title{
Towards A Semantic Tagger for Analysing Contents of Chinese Corporate Reports
}

\author{
Scott Piao ${ }^{12}$ \\ School of Computing and Communications, Lancaster University \\ Lancaster LA1 4WA, United Kingdom \\ E-mail: s.piao@lancaster.ac.uk \\ Xiaopeng $\mathbf{H u}^{3}$ \\ China Center for Information Industry Development (CCID) \\ CCID Plaza, 66 Zizhuyuan Rd, Haidian District, Beijing, China \\ E-mail: huxp@ccidtrans.com
}

\section{Paul Rayson}

School of Computing and Communications, Lancaster University

Lancaster LA1 4WA. United Kingdom

E-mail: p.raysonelancaster.ac.uk

\begin{abstract}
In this paper, we report on an experiment in which we explore the feasibility of applying a semantic tagger to analyze the textual contents of Chinese corporate reports andfocuse on the contents of corporate strategy. In recent years, Natural Language Processing (NLP) research has paid increasing attentions to the automatic analysis of the textual contents of corporate reports by using NLP approach on a large scale. We test the assumption that the semantic annotation tools can be useful for such a purpose and study the feasibility by testing a Chinese semantic tagger developed by UCREL, Lancaster University for extracting core Chinese terms and semantic concepts from Chinese corporate annual disclosures, focusing on three main USAS semantic categories, Money \& Commerce, Architecture \& Buildings, and Science \& Technology, which we assume are closely related to the corporate strategy description, and use these categories and tags to extract core strategy terms. Our study shows that, by carefully applying the selected semantic categories, our semantic annotation tool is capable of extracting core Chinese terms which can further facilitate the content analysis of Chinese corporate reports.
\end{abstract}

ISCC 2015

18-19, December 2015

Guangzhou, China

\footnotetext{
${ }^{1}$ Corresponding Author

${ }^{2}$ This study is supported by NSFC Dean's award 2016 of China, UCREL of Lancaster University, UK and The China Center for Information Industry Development (CCID), Beijing, China.

${ }^{3}$ Speaker
} 


\section{Introduction}

Recent Natural Language Processing (NLP) research has paid increasing attention to the automatic analysis of the textual contents of corporate business reports on a large scale, such as in Big Data contextso as to provide useful information for stakeholders such as investors, regulators and governments who are concerned with the performance of commercial companies.

The importance of analysis of corporate reports has long been recognised in business and economic areas, and a substantial amount of researches have been carried out in this regard. For example, Jones and Shoemaker analyzed a collection of accounting narrative papers for the types of linguistic features and readability [1]. Rutherford analyzed the UK Operating and Financial Review as a genre of accounting narrative, and applied a word-frequency based corpus linguistics approach to identify the genre rules [2]. Beattie and Thomson investigated the issue of applying the content analysis approach to the analysis of intellectual capital disclosures [3]. More recently, Brennan et al. investigated the issue of impression management in accounting communication through the analysis of accounting narratives [4], Davies et al. examined typology of text analysis approaches in corporate narrative reporting research [5], and Hoberg and Lewis applied linguistic analysis and statistical metrics to detect the fraud corporate disclosures [6]; however, majority of the above-mentioned studies were based on small sets of data or manual analysis. With the increasing amounts of corporate reports such analysis has to be automated for applciation on a large scale.

A recent new development refers to the application of the NLP approach to facilitate the automatic content analysis of corporate business reports on a large scale. For example, El-Haj et al. developed techniques and a tool to automatically parse and annotate document structures of the UK corporate reports [7]. Young suggested that NLP techniques can be used to address various issues of the corporate communications including 1) detecting deception and fraudulent reporting; 2) measuring sentiment (market and individual stock; 3) opinion mining; 4) measuring information content of the corporate narratives, etc. [8]

So far, the majority of researches on automatic textual content analysis of corporate reports have been conducted on English data. Similar researches on Chinese data are still very limited. Our research aims at addressing this gap and developing a semantic analysis tool for automatically analyzing corporate business reports made in Chinese. Specifically, in this experiment, we focus on the issue of automatic identification and extraction of core Chinese terms and concepts which can be used to search for business-strategy related information in a large collection of Chinese corporate business reports.

\section{USAS Semantic Annotation System}

In our study, we use a Chinese semantic tagger developed by UCREL, Lancaster University, UK to automatically identify core Chinese terms related to the corporate strategy. It is a part of the Lancaster USAS semantic annotation software system which is based on a set of semantic lexicons and applies a set of coarse-grained word sense disambiguation rules [9]. This system employs a semantic classification scheme derived from Tom McArthur's Longman Lexicon of Contemporary English [10], as well as a set of tags for denoting the semantic fields of the classification scheme. In detail, the USAS semantic scheme contains 21 main semantic fields which are denoted by 21 letters. They are further divided into 232 sub-fields, such as $I 1.2$ 
for "Money: Debts", K5.1 for "Sports", N5 for "Quantities" etc.(For further details about USAS system, see http://ucrel.lancs.ac.uk/usas/). In addition to the formal tagset, it also employs a set of auxiliary codes, such as $\boldsymbol{m} / \boldsymbol{f}$ (male/female), +/- (positive/negative) etc. to distinguish important attributes of semantic fields. For example, the antonyms "happy" and "sad" are tagged with "E4.1+" and "E4.1-", indicating positive and negative sentiment respectively; furthermore, it is designed to identify and tag the multi-word expressions as single terms, such as phrasal verbs, noun phrases, named entities and non-compositional idioms, which is highly significant for identifying the contextual meaning.

Originally developed for processing English textual data, it has been ported to a number of other languages, including Chinese [11]. The current version of Chinese semantic tagger is capable of annotating Chinese words by using the USAS semantic tagset with a good lexical coverage. Although it is still under improvement, we hypothesise that it is suitable for our study, where we only focus on three main USAS semantic categories.

The Chinese semantic tagger software incorporates a Chinese word segmenter and a POS tagger developed in Stanford University (http://nlp.stanford.edu/software/tagger.shtml), based on which, the Chinese semantic package carries out semantic annotation. It employs Chinese semantic lexicons derived from the English semantic lexicons via automatic translation by using a Chinese-English bilingual dictionary and a corpus-derived bilingual lexicon [11].

\section{Experiment of Corporate Strategy Related Core Term Extraction}

As mentioned previously, we aim at examining the feasibility of identifying core Chinese terms in the Chinese corporate reports that are related to the description of corporate business strategy. A collection of such terms can help generate the macro summaries regarding the corporate strategies reflected by their business reports as well as provide data searching points for further analysis. Obviously, not all semantic fields are related to the business strategy information; therefore, for our experiment, we chose three main USAS semantic fields which we consider are closely relevant to the strategic contents in the corporate reports. The three selected fields (denoted by three letters) include:

1) I-Money \& Commerce,

2) H-Architecture, Buildings, Houses \& Home,

3) $Y$ - Science \& Technology.

In the USAS semantic annotation scheme, the above three categories are further divided into eighteen sub-categories (tags), as shown in Table 1 below. As indicated by their definitions, the categories under the $I$ major category are closely related to financial and commercial entities and activities. In addition, those under the $H$ major category can cover terms related to corporate office buildings and factories etc. while those under the $Y$ major category can cover terms related to the research and development activities in corporates; therefore, we assumed that these three USAS semantic major fields can be useful in identifying and extracting core Chinese terms related to corporate entities, events and strategy. In practice, we collected and analyzed the Chinese terms annotated by these USAS tags listed in Table 1 and investigate to what extent our approach can facilitate the automatic semantic content analysis of the corporate reports. 


\begin{tabular}{|c|c|c|}
\hline$I$ & $\boldsymbol{H}$ & $\boldsymbol{Y}$ \\
\hline $\begin{array}{l}\text { I1 - Money generally } \\
\text { I1.1 - Money: Affluence } \\
\text { I1.2 - Money: Debts } \\
\text { I1.3 - Money: Price } \\
\text { I2 - Business } \\
\text { I2.1 - Business: Generally } \\
\text { I2.2 - Business: Selling } \\
\text { I3 - Work and employment } \\
\text { I3.1 - Work and employment: } \\
\quad \text { Generally } \\
\text { I3.2 - Work and employment: } \\
\quad \text { Professionalism } \\
\text { I4 - Industry }\end{array}$ & $\begin{array}{l}H 1 \text { - Architecture, kinds of } \\
\quad \text { houses \& buildings } \\
\text { H2 - Parts of buildings } \\
\text { H3 - Areas around or near houses } \\
\text { H4 - Residence } \\
\text { H5 - Furniture and household } \\
\quad \text { fittings }\end{array}$ & $\begin{array}{l}Y 1 \text { - Science and technology in } \\
\text { general } \\
Y 2 \text { - Information technology and } \\
\text { computing }\end{array}$ \\
\hline
\end{tabular}

Table 1: USAS Semantic Fields/Tags under Three Main Categories $I, H$ and $Y$.

\subsection{Collection of Corporate Business Reports as Test Data}

For the test data of our experiment, we collected some business annual reports from a central Chinese corporation website (http://www.nbdqw.com/), where the publically accessible annual business disclosure reports of Chinese corporates are listed. Such annual reports publicize the corporates' performances and achievements in the earlier year as well as announcement of their future plans and strategies for business promotion purposes. This type of data provides valuable resources for analysis and prediction of thecorporate information via the NLP approach. In order to guarantee a wide representativeness and a high quality of data testing, we manually selected annual disclosure reports of ten represenative Chinese public companies published from 2007 to 2014 wherever available (pseudonyms are used for the companies in this paper). All together, we collected 57 reports containing 3,584,956 Chinese characters. In terms of the industry area, they had a wide coverage of China's industry, spanning high technology sector, building and construction, automobile production, travel and tourism, chemical industry, heavy industry, electronics industry and petroleum industry, etc.. We used the sample reports to study and test our methodology to automatically extract information about business strategy of different types of corporates. In this particular experiment, we used the sample data to test our method for automatically identifying core Chinese terms that were related to the business strategy. In order to estimate the lexical coverage of the semantic tagger for the corporate data, we calculated the percentage of the Chinese words in the sample reports that are recognised by our semantic tagger. Table 2 below shows a breakdown of test of data distribution and Chinese semantic tagger lexical coverage for the ten corporates' reports, where the columns represent individual corporates and the first, second and third rows list respectively the number of documents, the size of documents in terms of Chinese characters and the the lexical coverage.

\begin{tabular}{|l|c|c|c|c|c|c|c|c|c|c|c|}
\hline & corp. 1 & corp. 2 & corp. 3 & corp. 4 & corp. 5 & corp. 6 & corp. 7 & corp. 8 & corp. 9 & corp. 10 & total \\
\hline docs num & 5 & 4 & 7 & 5 & 6 & 6 & 6 & 7 & 6 & 5 & 57 \\
\hline chi. chars & 336,133 & 251,686 & 245,275 & 264,416 & 342,622 & 276,025 & 570,537 & 593,088 & 422,743 & 282,431 & $3,584,956$ \\
\hline lex cov. & $76.91 \%$ & $75.41 \%$ & $77.08 \%$ & $75.57 \%$ & $78.26 \%$ & $75.95 \%$ & $74.86 \%$ & $75.57 \%$ & $74.42 \%$ & $78.16 \%$ & $76.02 \%$ \\
\hline
\end{tabular}

Table 2: Breakdown of Tested Data Size and Lexical Coverage of Chinese Semantic Tagger for Annual Disclosure Reports from Rach Corporate. 
As these corporate reports were published as PDF documents, we had to extract the text data from them in order to apply our semantic tagger software. We used Foxit PDF reader software (https://www.foxitsoftware.com/) for extracting the textual contents of the reports into plain text files. It is well known that it is highly challenging to extract clean text data from PDF documents, and inevitably our extracted text files contain some broken lines and words. Assuming such noise do not significantly affect the results of our experiment, we only carried out minor noise cleaning process such as removing number matrices derived from tables and page breaking lines etc.

\subsection{Data Processing, Core Term Extraction and Manual Rating}

The text files extracted from the PDF documents were processed by using the Chinese semantic tagger. The reports were processed separately for each corporate in order to investigate how the different types of corporate business influence the results of our approach. For each corporate, we separately collected the terms which were tagged with USAS tags falling under the three main USAS semantic fields $I, H$ and $Y$ (refer to Table 1). Next, we collected the frequencies of the terms along with their tags (e.g. 资产_H1/H3), and then selected the most frequent 100 term_tag pairs for each corporate and for each of the three main semantic fields. As a result, we obtained three term-tag frequency lists for each corporate, which contained entries in the format shown below (the brackets are for inputing manual raking scores later).

$\begin{array}{lll}\text { \#Freq. } & \text { Word_Tag } & \text { Manual Rating } \\ 2866 & \text { 资产_H1/H3 } & \text { [ ] } \\ 1226 & \text { 合并_H2 } & \text { [ ] }\end{array}$

Finally we asked human raters who wer familiar with corporate business reports to rank the terms by using a numerical scale and give a score to each term by using the guidelines shown in Table 3.

\begin{tabular}{|l|l|}
\hline rating scale & Description of rating criteria \\
\hline 5 & Closely related to corporate strategy description, e.g. 资产, 合并. \\
\hline 4 & Fairly related to corporate strategy description, e.g. 方法, 计划 etc. \\
\hline 3 & Ordinary nouns, verbs, e.g. 日录, 计算. \\
\hline 2 & Meaningful single character words, such as measurement 层. \\
\hline 1 & Irrelevant words. \\
\hline
\end{tabular}

Table 4: Description of Rating Criteria for Human Raters.

According to the above rating scale criteria, the rating score of 3 indicates neutral terms. Therefore, if a term is rated with scores of 5 and 4 , it is considered to be relevant to the corporate strategy information with a certain degree. Furthermore, if a group of terms have an average rating score above 3 , it indicates that the terms collectively carry certain information about corporate strategy. The higher the scoreis, the more strategy information they carry. Following this criteria, in our experiment we measured performance of a method by observing the average rating score obtained by the terms extracted by it, with an average score above 3 indicating certain level of success, and with the average score of 5 indicating the maximum success. 


\subsection{Evaluation}

Based on the human rater's scores, we assessed the performance of our approach in terms of the effectiveness of each USAS semantic category for the corporate strategy related term extraction. In detail, for each company, we calculated the average human raters' raking score for the $I, H$ and $Y$ semantic categories respectively in order to assess to what extent these terms falling under these categories carried corporate strategy related information. Table 4 below shows the results, where the columns represent the ten Chinese corporates and the rows represent the three major semantic categories.

\begin{tabular}{|c|c|c|c|c|c|c|c|c|c|c|c|}
\hline $\begin{array}{c}\text { sem } \\
\text { cat }\end{array}$ & corp. 1 & corp. 2 & corp. 3 & corp. 4 & corp. 5 & corp. 6 & corp. 7 & corp. 8 & corp. 9 & $\begin{array}{c}\text { corp. } \\
10\end{array}$ & avg \\
\hline I & 4.33 & 3.52 & 3.66 & 3.8 & 4.18 & 3.44 & 3.27 & 3.32 & 3.28 & 3.25 & 3.60 \\
\hline H & 3.43 & 3.07 & 3.02 & 3.15 & 3.06 & 3.06 & 3.28 & 3.07 & 3.4 & 3.28 & 3.18 \\
\hline Y & 3.13 & 2.9 & 3.04 & 3.53 & 3.07 & 2.83 & 2.83 & 2.93 & 2.97 & 2.92 & 3.01 \\
\hline
\end{tabular}

Table 4: Statistics of Manual Rating of Terms for Ten Company Reports.

As shown in table 4, the $I$ semantic category produced the best average results, reflected by the average rating score of 3.60; on the other hand, the $Y$ category produced the worst result, obtaining scores below 3 for six companies, indicating many terms extracted by using this category are irrelevant to the corporate strategy information. Given that the rating score of 3.0 indicates the neutral terms, the average scores greater than 3.0 imply many of the extracted terms bear certain information about the corporate strategy information. We observed that some broken words caused by errors in the pdf-to-text conversion and Chinese word segmenting processes included in the term lists and we suspected they might affect the results; therefore, we filtered out the single-character terms and re-calculated the statistics of the human rating. Table 5 below shows the results.

\begin{tabular}{|c|l|l|l|l|l|l|l|l|l|l|l|}
\hline $\begin{array}{c}\text { sem } \\
\text { cat }\end{array}$ & corp. 1 & corp. 2 & corp. 3 & corp. 4 & corp. 5 & corp. 6 & corp. 7 & corp. 8 & corp. 9 & corp. 10 & avg \\
\hline I & 4.5 & 3.65 & 3.76 & 3.88 & 4.30 & 3.55 & 3.40 & 3.45 & 3.41 & 3.38 & 3.73 \\
\hline H & 3.89 & 3.48 & 3.44 & 3.51 & 3.38 & 3.37 & 3.31 & 3.29 & 3.77 & 3.61 & 3.50 \\
\hline Y & 3.45 & 3.30 & 3.33 & 3.83 & 3.45 & 3.17 & 3.15 & 3.21 & 3.15 & 3.18 & 3.32 \\
\hline
\end{tabular}

Table 5: Statistics of Manual Rating of Terms after Removing Single Character Words.

The comparison between Table 4 and Table 5 reveals that the broken words indeed affected the results. For example, by removing the Chinese single-character words, many of whom derived from broken words, the average rating score of $I$ category was improved by 0.13 . Figs. 1, 2 and 3 illustrate the improvements achieved by the filtering, where points represent the ten companies and the orange and grey lines represent respectively the average rating scales for the three main semantic categories obtained with and without single character words included. These graphs demonstrate a consistent noticeable improvement of the rating scores after the filtering except for Company 7 (building and construction) in $H$ category. Overall, the filtering has a significant impact on $H$ and $Y$ categories. This result indicates that Chinese singlecharacter words may not be suitable for the key term extraction in the content analysis of corporate reports. 


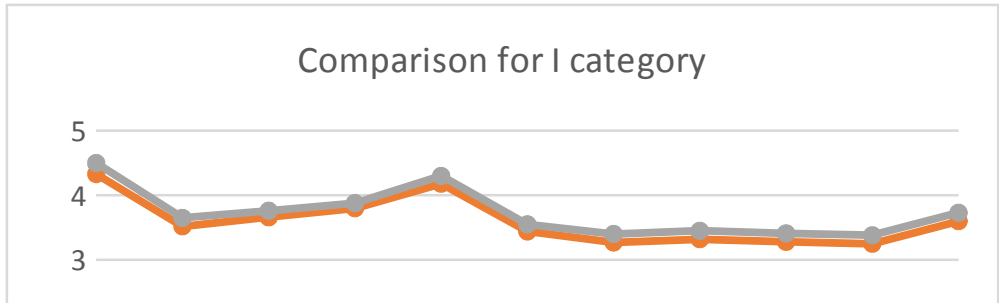

Figure 1: Rating Scores Comparison for $I$ category.

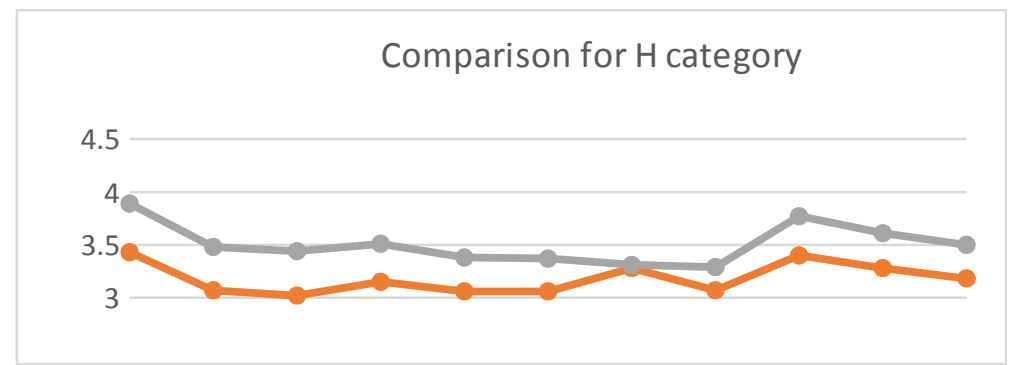

Figure 2: Rating Scores Comparison for $H$ Category.

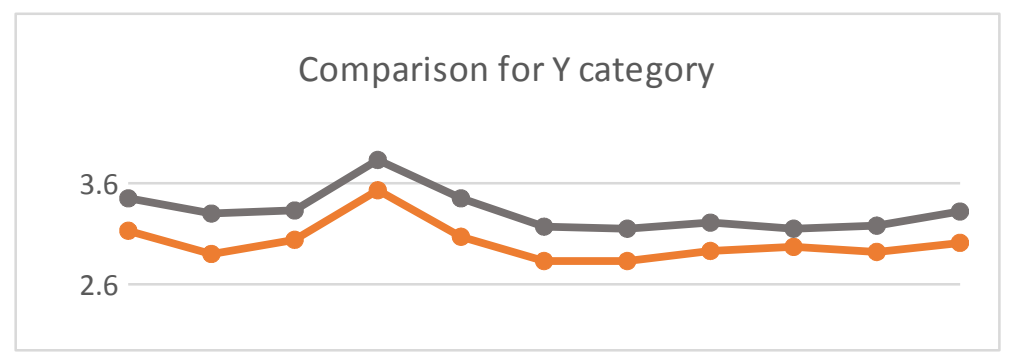

Figure 3: Rating Scores Comparison for $Y$ Category.

Although limited and preliminary, our experiment and the analysis of results show that, with carefully selected semantic categories and proper text cleaning process, the Chinese semantic tagger can potentially facilitate rapid automatic extraction of corporate strategy related terms and concepts, which is crucial for achieving timely delivery of corporate business information to stakeholders and clients based in large-scale data, such as in big data context. As far as we know our experiment is the first study on automatic extraction of corporate strategy related Chinese terms, it is difficult to make direct comparison with other existing methods. With the development of our researches, standard test data will be produced and more methods will be tested in order to find an optimal solution.

\section{Conclusion}

In this paper, we have reported our experiment in which we tested the feasibility of automatically identifying and extracting corporate strategy related Chinese terms from corporate annual disclosure documents by using a Chinese semantic tagger. The analysis of our experiment results demonstrates that it is feasible to use such a tool to automatically extract key Chinese terms for further analysis of corporate strategy information. In future work, we will improve the lexical coverage and accuracy of the semantic tagger and design a better approach to select appropriate semantic tags to improve the analysis of corporate reports. 


\section{References}

[1] M.J. Jones and P.A. Shoemaker. Accounting narratives: a review of empirical studies of content and readability. Journal of Accounting Literature, 13, 142-85 (1994)

[2] B. A. Rutherford. Genre analysis of corporate annual report narratives - a corpus linguistics-based approach. Journal of Business Communication, Volume 42, Number 4, pp. 349-378 (2005)

[3] V. Beattie and S. Thomson. Lifting the lid on the use of content analysis to nvestigate intellectual capital disclosures. Accounting Forum, 31(2),129-163 (2007)

[4] N. M. Brennan and D. M.Merkl-Davies. Accounting narratives and impression management. In: Russell J. Craig, Jane Davison and Lisa Jack (eds.), The Routledge Companion to Accounting Communication. London: Routledge, pp.109-132 (2013)

[5] D. M. Merkl-Davies, N. M. Brennan and P. Vourvachis. Content analysis and discourse analysis in corporate narrative reporting research: a methodological guide. Centre for Impression Management in Accounting Communication (CIMAC) Conference 2014. Bangor, UK. (2014)

[6] G. Hoberg and M. L. Craig. Do fraudulent firms strategically manage disclosure?. The 8th LSE/LUMS/MBS Conference, ICAEW, London. (2014)

[7] M. El-Haj, P. Rayson, S. Young and M. Walker. Detecting document structure in a very large corpus of UK financial reports. In Proceedings of The 9th Edition of the Language Resources and Evaluation Conference, Reykjavik, Iceland. (2014)

[8] S. Young. Textual analysis and investment decisions: an overview. Sell-Side Meeting: Citi Investment Research \& Analytics, 2014 Citi Quantitative Finance Conference. Valencia, Spain. (2014)

[9] P. Rayson, D. Archer, S. Piao and T. McEnery. The UCREL semantic analysis system. In proceedings of the workshop on Beyond Named Entity Recognition Semantic labelling for NLP tasks in association with 4th International Conference on Language Resources and Evaluation (LREC 2004), Lisbon, Portugal, pp. 7-12 (2004)

[10] T. McArthur. Longman Lexicon of Contemporary English. Longman London. (1981)

[11] S. Piao, F. Bianchi, C. Dayrell, A. D'Egidio and P. Rayson. Development of the multilingual semantic annotation system. In Proceedings of The 2015 Conference of the North American Chapter of the Association for Computational Linguistics - Human Language Technologies (NAACL HLT 2015), Denver, Colorado, USA. (2015) 